\title{
Primary rectal lymphoma: A case report and literature review
}

\author{
CHUNHUI JIANG, LEI GU, MENG LUO, QING XU and HONG ZHOU \\ Department of Gastrointestinal Surgery, Renji Hospital, Shanghai Jiaotong University School of Medicine, \\ Pudong, Shanghai 200001, P.R. China
}

Received June 5, 2014; Accepted February 19, 2015

DOI: $10.3892 / \mathrm{ol} .2015 .3164$

\begin{abstract}
One-third of non-Hodgkin's lymphoma cases are extranodal lymphomas. The gastrointestinal tract is the most common of the extranodal sites. The incidence of primary rectal lymphoma in the gastrointestinal tract is very rare, and its clinical presentation is difficult to distinguish from that of rectal carcinoma. In the treatment of primary rectal lymphomas, surgery options have been controversial. Considering this, we report a case of primary rectal lymphoma (diffuse large B-cell lymphoma) which disappeared completely following cyclophosphamide, hydroxydaunomycin, oncovin and prednisolone chemotherapy. This case demonstrates that systemic chemotherapy may be successful for the primary treatment of rectal lymphoma.
\end{abstract}

\section{Introduction}

Lymphomas are malignant lymphocytes. They may be classified as Hodgkin's lymphoma and non-Hodgkin's lymphoma (1). Non-Hodgkin's malignant lymphomas may be divided into nodal and extranodal lymphoma. The gastrointestinal tract is the most common site of extranodal lymphoma (2).

Primary gastrointestinal lymphoma occurs in the absence of evidence of systemic disease. Dawson et al (3) established criteria for the diagnosis of primary colorectal lymphomas in 1961. These are: No palpable, superficial lymph nodes at presentation; no enlarged mediastinal lymph nodes on chest $\mathrm{X}$-ray; normal range for white blood cell count including total and differential; at surgery, only the regional lymph nodes are involved; and the liver and spleen are without disease.

Controversy remains with regard to the therapeutic approaches of treating gastrointestinal lymphomas. The optimal treatment for primary rectal lymphoma remains unclear, and further research is still required. In this paper, we present a case of primary lymphoma of the rectum and focus on the role of chemotherapy.

Correspondence to: Mr. Hong Zhou, Department of Gastrointestinal Surgery, Renji Hospital, Shanghai Jiaotong University School of Medicine, 1630 Dongfang Road, Pudong, Shanghai 200001, P.R. China

E-mail: renjizhouhong@163.com

Key words: primary, rectal lymphoma, chemotherapy

\section{Case report}

In July 2012, a 51-year-old female attended the outpatient clinic of Renji Hospital, Shanghai, China, with complaints of change in bowel habits, rectal bleeding. Rectoscopy revealed proctitis with erosion, and the histology of biopsy specimens revealed chronic inflammation of mucous membranes.

When the patient came to our clinic, her clinical manifestation was severe rectal bleeding. A palpable mass was identified on directeral rectal examination. The mass was located in the dentate line, and fixed to the rectal wall. The superior border of the tumor could not be reached. A repeat colonoscopy revealed a three-quarters circumferential lesion approximately $3-8 \mathrm{~cm}$ from the anal verge. The ulcer was infiltrating with necrotic tissue in the central area, and the margins demonstrated nodular uplift (Fig. 1). By histopathological diagnosis, the rectal biopsy specimen was diagnosed as non-Hodgkin's diffuse large B-cell lymphoma (DLBCL). The results of immunohistochemical staining were CD20(+), CD79a(+), CD30(+), Bcl-2(-), CD10(-), Mum-1(-), CD21(-), CD23(-), CK(-), Vim(+) and $\mathrm{Ki}-67>40 \%$. Abdominopelvic computed tomography (CT) demonstrated that the rectal wall had local thickening, and the fat gap surrounding the colon wall contained small scattered lymph nodes (Fig. 2). Additional examinations did not reveal any evidence of infiltration to other organs.

It was finally decided not to resect the rectum where the lesion located due to the fact that the lesion completely disappeared after the patient had received four cycles of cyclophosphamide, hydroxydaunomycin, oncovin and prednisolone (CHOP) chemotherapy. A complete remission was achieved and confirmed by abdominal CT scan and colonoscopy with a biopsy of the diseased region.

\section{Discussion}

Primary rectal lymphoma is the rarest disorder of all primary gastrointestinal lymphomas, accounting for $0.1-0.6 \%$ of all colonic malignancies and $0.05 \%$ of all primary rectal malignancies (4). The majority of patients with rectal lymphoma present with non-specific symptoms or negative rectal biopsy, which often leads to delays in diagnosis and an advanced stage at presentation, where rectal bleeding may be observed as with primary rectal carcinoma (5). Endoscopy with biopsy and abdominal CT are the most valuable diagnostic tests.

CT examination for the diagnosis of rectal lymphoma has not attracted sufficient attention for a long time. Primary colorectal 


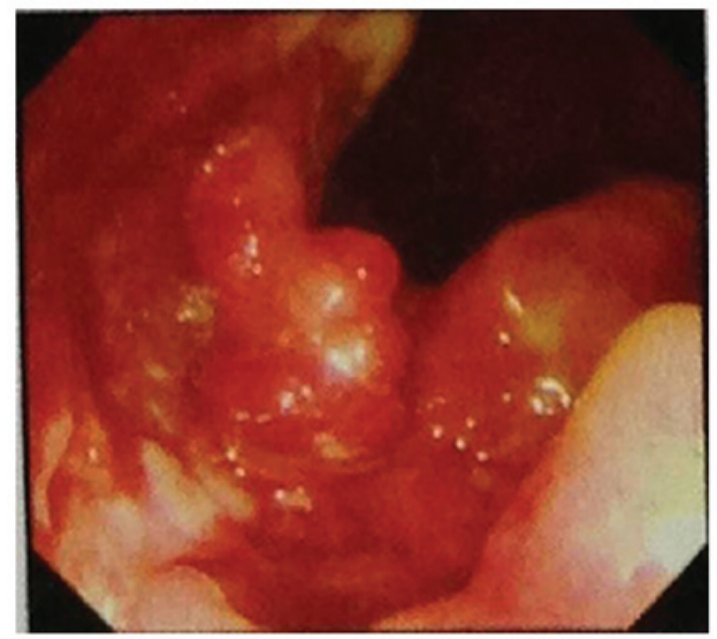

Figure 1. Appearance of the lesion revealed by colonoscopy.

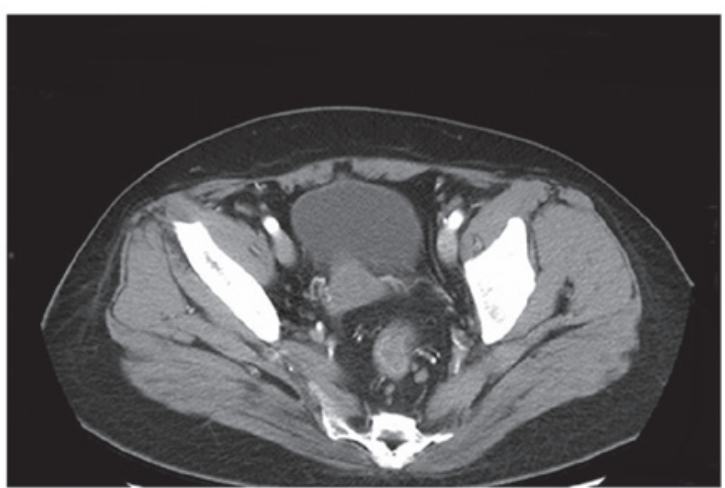

Figure 2. Abdominopelvic computed tomography image of the lesion.

lymphomas often manifest as discrete masses and tend to have a greater depth of mural invasion than infiltrative lesions (6). Research has identified that when CT reveals concentric wall thickening, involving the full thickness of the bowel wall without an associated desmoplastic reaction, lymphoma should be the primary consideration in the differential diagnosis $(7,8)$. Although the characteristic tumor morphology and imaging aid in the diagnosis, pathological analysis of a biopsy remains the gold standard for diagnosis of this disease. As colorectal lymphoma originates from the submucosa of the colon, accurately obtaining a biopsy sample may be challenging, and the pathological positive rate is low $(9,10)$. Therefore when a biopsy is obtained via colonoscopy, experienced endoscopists are required. As there is only a low incidence of the disease, there have been no prospective studies evaluating the relative benefits of adjuvant chemotherapy, radiotherapy, surgery or a combination of treatments for primary rectal lymphoma (11). DLBCL of the colorectum generally has a uniform method of treatment: chemotherapy followed by aggressive surgical treatment (12). Due to its varied histological behaviors and clinical presentations, rectal lymphoma should be considered as a different clinicopathological type. For this reason, individualized treatment programs need to be developed based on specific clinical situations (13). Although it is not possible to make a reasonable conclusion with a few cases, we emphasize the significance of chemotherapy in achieving a successful cure for patients with rectal DLBCL.

\section{References}

1. Jaffe E, Harris NL, Stein H, Vardiman JW (eds): WHO Classification of Tumours: Pathology and Genetics of Tumours of Hematopoietic and Lymphoid Tissues. IARC Press, Lyon, 2001.

2. Muller-Hermelink HK, Chott A, Gascoyne RD and Wotherspoon A: B-cell lymphoma of the colon and rectum. In: WHO Classification of Tumours. Pathology and Genetics of Tumours of the Digestive System. Hamilton SR and Aaltonen LA (eds). IARC Press, Lyon, pp139-141, 2000.

3. Dawson IM, Cornes JS and Morson BC: Primary malignant lymphoid tumours of the intestinal tract. Report of 37 cases with a study of factors influencing prognosis. Br J Surg 49: 80-89, 1961.

4. Dodd GD: Lymphoma of the hollow abdominal viscera. Radiol Clin North Am 28: 771-783, 1990.

5. Quayle FJ and Lowney JK: Colorectal lymphoma. Clin Colon Rectal Surg 19: 49-53, 2006.

6. Wyatt SH, Fishman EK, Hruban RH and Siegelman SS: CT of primary colonic lymphoma. Clin Imaging 18: 131-141, 1994.

7. Degeorges S, Mesnil A, Marion-Audibert AM, et al: Ano-rectal symptoms, related to Epstein-Barr Virus-Associated Burkitt's lymphoma in an immunocompetent patient. Gastronterol Clin Biol 31: 442-444, 2007 (In French).

8. Stanojevic GZ, Nestorovic MD, Brankovic BR, et al: Primary colorectal lymphoma: An overview. World J Gastrointest Oncol 3: 14-18, 2011.

9. Zhang RP, Qiang L, Tao S, et al: 68 cases of primary colorectal non-Hodgkin's lymphoma clinical analysis. Zhongguo Zhonghua Weichang Waike Zazhi 6: 49-51, 2004 (In Chinese).

10. Yang JO, Zhang S, Zhen WJ and Zhen HH: Diagnosis and treatment of primary malignant lymphoma of the rectum. Jie Zhichang Gangmen Waike 18: 13-15, 2012 (In Chinese).

11. Friedlich M, Lamba M and Stern H: Management of rectal lymphoma. Can J Surg 47: 142 -143, 2004.

12. Avilés A, Neri N and Huerta-Guzmán J: Large bowel lymphoma: an analysis of prognostic factors and therapy in 53 patients. J Surg Oncol 80: 111-115, 2002.

13. Bilsel Y, Balik E, Yamaner S and Bugra D: Clinical and therapeutic considerations of rectal lymphoma: a case report and literature review. World J Gastroenterol 11: 460-461, 2005. 